\title{
Determinación de los principales factores que influyen en las lesiones músculo esqueléticas de los trabajores del Mercado Central de Cartago, Costa Rica
} Factors that influence in musculoskeletal injuries in the premises of the Central Market of Cartago, Costa Rica

Ara Villalobos Rodríguez ${ }^{1}$, Miriam Brenes-Cerdas²

Villalobos-Rodríguez, A; Brenes-Cerdas, M. Determinación
de los principales factores que influyen en las lesiones mús-
culo esqueléticas de los trabajores del Mercado Central de
Cartago, Costa Rica. Tecnología en Marcha. Vol. 33-3. Julio-
Setiembre 2020. Pág 105-116.

doi) https://doi.org/10.18845/tm.v33i3.4469

Fecha de recepción: 24 de julio de 2019

Fecha de aprobación: 6 de noviembre de 2019

1 Ingeniera. Instituto Tecnológico de Costa Rica, Escuela de Ingeniería en Seguridad Laboral e Higiene Ambiental, Costa Rica. Correo electrónico: avillalobos@tec.ac.cr (D) https://orcid.org/0000-0003-3340-8136

2 Ingeniera. Instituto Tecnológico de Costa Rica, Escuela de Ingeniería en Seguridad Laboral e Higiene Ambiental, Costa Rica. Correo electrónico: mibrenes@tec.ac.cr (iD https://orcid.org/0000-0001-8850-8359 


\section{Palabras clave}

Mercado; factores músculo esqueléticos; manejo manual de cargas; riesgos.

\section{Resumen}

Las lesiones músculo-esqueléticas tienen un costo considerable para el sistema de salud pública [1], es por ello que el objetivo del proyecto fue contribuir al mejoramiento del ambiente de trabajo en las pequeñas y medianas empresas (PYME), por medio de la determinación de los factores que influyen en las lesiones músculo-esqueléticas en las operaciones de manejo manual de cargas. Para ello se hizo uso del método MAC y de equipos de medición como los dinamómetros y relojes polar. Factores como el peso de las cargas, el traslado de éstas a lo largo de distancias mayores a los 150 m, además de la forma y tamaño de las mismas, aumentan la frecuencia cardiaca en los trabajadores. Los resultados obtenidos evidencian que las tareas de transporte, levantamiento y acomodo de los productos, son clasificadas como riesgo crítico, debido a que son realizadas de forma incorrecta por los trabajadores; siendo el principal riesgo el biomecánico, debido a peligros como las posturas inadecuadas y prolongadas, manejo de cargas por espacios reducidos y en locales con dimensiones insuficientes, aunados a las características personales como la escaza actividad física, y la forma en las que se realizan las tareas, en donde predomina el esfuerzo muscular dinámico. Esta información es la base para que, a nivel de locales del Mercado Central de Cartago se realice una evaluación de riesgos a detalle, que permita adoptar medidas necesarias en lo que concierne a la formación de métodos de trabajo y medios técnicos apropiados en el transporte y manejo de carga.

\section{Keywords}

Market; musculoskeletal factors; manual handling of heavy loads; risks.

\section{Abstract}

The muscular- skeletal injuries have a considerable cost for the public health[1], which is why the objective of the project was to contribute to the improvement of the work environment in the small and medium-sized enterprises, through the determination of the factors that influence in the muscular-skeletal injuries in manual handling of heavy loads operations. For it, the MAC method and measurement equipment like dynamometers and Polar watches were used to determine if the weight, distance, shape and size of the loads were a risk factor.

The results reveal that the tasks of transportation, lifting and accommodation of the products were classified as critical risk, caused by incorrectly handling, the main risk detected was the biomechanical, derived of inadequate and prolonged positions, narrow corridors and small establishments; this added to the personal characteristics like low physical activity, and in the way the tasks are done wherein predominate the dynamic muscular effort. With this information the Central Market of Cartago, can make a risks evaluation in detail, also allow to adopt necessary measures regarding the formation of working methods and technical means appropriate in transport and load handling. 


\section{Introducción}

En los últimos años los gobiernos de América Latina y el Caribe han impulsado a las PYME por medio de programas de apoyo, lo que ha mejorado el desempeño económico y productivo de las mismas [2], sin embargo, las iniciativas desarrolladas en la mayoría de los casos no consideran el aspecto de la salud laboral.

Las lesiones músculo esqueléticas se han convertido en uno de los principales problemas de salud en el trabajo y causa de ausentismo laboral, con repercusiones económicas importantes para el trabajador, la empresa y la sociedad en general [3]; [4] además de una disminución en la productividad, alteración en la calidad de vida del trabajador, entre otras consecuencias [5]. El principal problema asociado a estas lesiones son las lumbalgias, producto de las tareas relacionadas con el levantamiento manual de cargas [6]; [7].

Además de las lumbalgias, los transtornos músculo esqueléticos se relacionan con una amplia gama de enfermedades que pueden provocar deterioro funcional de otras zonas del cuerpo, como el cuello, hombros, muñecas, manos [8].

Los trastornos músculo-esqueléticos, según el informe de la Agencia Europea para la Seguridad y Salud en el Trabajo, son el problema de salud laboral más común en Europa [9]. Situación no ajena a nuestro país, en donde de acuerdo al informe proporcionado por el Ministerio de Trabajo y Seguridad Social [10], los accidentes por sobrecarga física ocupan el segundo lugar, correspondientes a 18.108 accidentes, que representan el $16 \%$ del total, fundamentalmente por sobreesfuerzos, manejo manual de cargas y trabajo dinámico.

Con el objetivo de evaluar los principales factores que influyen en las lesiones múscloesqueléticas, se analizaron las actividades concernientes al manejo manual de cargas, que se efectúan en empresas PYME, específicamente las que se desarrollan por un grupo de trabajadores del Mercado Central de Cartago, debido a que este tipo de tarea está presente en la mayoría de las actividades que en dicho centro de trabajo se realizan, además que el entorno en donde se desarrollan las mismas, en la mayoría de las ocasiones es reducido, por lo que el riesgo aumenta.

Para alcanzar esos resultados se plantearon como objetivos el identificar las actividades realizadas por los trabajadores en las operaciones de manejo manual de cargas de los locales del mercado, que influyen en la ocurrencia de lesiones músculo esqueléticas, para posteriormente realizar la evaluación, y por último establecer recomendaciones generales dirigidas a la población en estudio.

\section{Materiales y métodos}

La población en estudio corresponde a los trabajadores del Mercado Central de Cartago (122 en total), la muestra varió, según la herramienta a aplicar. El estudio fue de tipo exploratorio en una muestra representativa seleccionada aleatoriamente, sobre la cual se aplicaron cuestionarios, con el fin de recibir retroalimentación de las actividades que realizan los trabajadores del mercado en las operaciones de manejo manual de cargas, también se efectuaron evaluaciones en las cuales se hizo uso de equipos que permitieron establecer la fuerza inicial y mantenida y el consumo metabólico, para determinar la intensidad del trabajo realizado.

\section{Entrevista}

Con el fin de identificar el peso de las cargas que los trabajadores levantan, en ella participaron 108 encargados de locales o sus representantes (los 14 restantes indicaron que no podían brindar ningún tipo de información, por motivos de confidencialidad). 


\section{Cuestionario}

Aplicado a 86 trabajadores (la muestra elegida fue no probabilística seleccionada a conveniencia, debido a que se consideró trabajar con 1 persona por cada local, que, según la información de la entrevista, levantaban cargas de más de 20kg, las cuales si se levantan con ambas manos la energía por kg y por metro de trabajo debe corresponder a un peso de 20 a $25 \mathrm{~kg}$ [11]. El objetivo de la aplicación de este instrumento era conocer más a detalle las actividades realizadas por los trabajadores en las operaciones de manejo manual de cargas, como lo es la información relacionada al tipo de labor, historial ocupacional y médico; con esto se pretendía conocer algunos de los factores de riesgo relacionados a la tarea y a las personas. Posterior a esta intervención se efectuaron reuniones en donde se invitaron a todas las personas que participaron del cuestionario, con el fin de explicarles a detalle las actividades que se iban a desarrollar en el proyecto, sin embargo, a las mismas solamente asistieron 14 personas que indicaron su anuencia a seguir colaborando, por lo que esta cantidad representa los locales a trabajar.

\section{Observación no participativa}

En donde se consideraron elementos como la técnica de levantamiento, formas, tamaños y materiales de las cargas, además de las condiciones del entorno de los locales (dimensiones, pasillos).

Evaluación de las operaciones que involucran un manejo manual de cargas que influyen en la ocurrencia de lesiones músculo esqueléticas

Para esto se hizo uso de los métodos que se comentan a continuación:

\section{Método MAC}

Previo al desarrollo de método, se aplicó una observación no participativa, para obtener información de la técnica de levantamiento, formas, tamaños y materiales de las cargas, además de las condiciones de la infraestructura, esto durante toda la jornada; tomando videos y fotografías de las actividades. Dicha metodología fue adaptada a las necesidades del proyecto (específicamente para operaciones de levantamiento y transporte), y se aplicó a 17 trabajadores (un trabajador para cada uno de los 14 locales participantes, sin embargo, en la carnicería se evaluaron 4 trabajadores, debido a que en el manejo manual de materiales que ellos realizan se ven comprometidas más partes del cuerpo), para posteriormente categorizar con base en los parámetros que establece el método, las actividades según los resultados alcanzados.

\section{Mediciones de variables}

Para el objetivo de evaluar las operaciones que involucran un manejo manual de cargas que podrían influir en la ocurrencia de lesiones músculo-esqueléticas; se trabajó con 17 trabajadores, a quienes se les realizaron mediciones (al final de la jornada) según la posición, para ambos brazos y manos con el dinamómetro, comparando los resultados con los valores según la edad, establecidos por el Programa de Terapia Ocupacional de la Universidad de Wisconsin- Milwaukee sobre los Datos Normativos para el agarre y la fuerza de pinza [12], y evaluación de la carga metabólica que se estableció a través de la técnica de medición de la frecuencia cardiaca promedio, gasto metabólico y edad de los trabajadores [13], mediante el uso de los datos obtenidos con el uso del reloj POLAR (modelo FT4).

\section{Evaluación de riesgos}

Se hizo uso de la Norma técnica INTE 31-06-07-2011 Guía para la identificación de los peligros y la evaluación de los riesgos de salud y seguridad ocupacional. 


\section{Resultados}

\section{Generalidades}

En la primera intervención (entrevista), se contó con la participación de dueños o encargados de 108 locales, en donde en el 80\% de ellos se manipulan pesos superiores a los $20 \mathrm{~kg}$, debido a que los pesos de 20 a $25 \mathrm{~kg}$ [11], resultan difíciles de manipular para la mayoría de las personas, convirtiéndose en un factor que influye en la ocurrencia de lesiones músculo esqueléticas. Con esta información se procedió a aplicar un cuestionario $(n=86)$, donde se obtiene que el $83 \%$ de la población son hombres; de ellos, la mayoría con edades en el rango de los 18 a 54 años, correspondiendo a un 82\%, el 18\% restante con 54 o más de 65 años, que, aunque representen el menor porcentaje, es un elemento importante a considerar, debido a que la probabilidad de sufrir trastornos dorsolumbares se incrementa con la edad, [14]. Es importante destacar que la capacidad en las facultades físicas (fortaleza muscular), en la tareas que exigen un alto nivel de destreza (como los ejecutados por los trabajadores del Mercado Central de Cartago), declina a medida que aumenta la edad [15], debido al deterioro sufrido por el organismo, producto del envejecimiento en personas mayores a 50 años [16], incluso de acuerdo a una investigación realizada [17], este es uno de los factores más influyentes en los problemas músculo esqueléticos.

En el cuestionario se consultó sobre la actividad física que realizan los trabajadores fuera de su jornada laboral, el 50\% de los entrevistados indicó que realiza ejercicios regularmente y de éstos, la mayoría practican algún tipo de ejercicio al menos una vez a la semana.

Con respecto a la frecuencia de transporte de cargas, de acuerdo a las personas entrevistadas la mayoría (79\%), realiza una manipulación de las mismas por menos de 30 minutos al día, lo que representa poco tiempo de la jornada laboral. Para los trabajadores que realizan manejo de cargas con una mayor frecuencia, se debe considerar que el resto del tiempo laboral es recomendable dedicarlo a actividades menos pesadas y que no impliquen la utilización de los mismos músculos, con el objetivo de que se logre su recuperación física [18].

Por ello se consultó a los participantes del cuestionario sobre las molestias que presentan actualmente en relación a su actividad laboral; de acuerdo a los resultados, la zona más afectada es la espalda dorsal, seguido del cuello, hombros, piernas y espalda lumbar respectivamente.

Como se indicó anteriormente, los trabajadores que participaron del cuestionario de dolencias manipulan pesos mayores a $20 \mathrm{Kg}$, y con base en las observaciones no participativas se determinó que el $100 \%$ de las personas no ejecutan la técnica correcta para el levantamiento manual de cargas, además las mismas son de diferentes tamaños (desde verduras en presentación individual hasta sacos de producto), materiales y formas, lo que en ocasiones dificulta la forma de agarre (como es el caso de las bandejas de los huevos, debido a que las estibas de cartón son inestables). Esta condición, en la forma y tamaño de las cargas, ocasiona que sean de difícil agarre, debido a que si la misma es muy ancha el colaborador se ve obligado a mantener posturas forzadas de los brazos, complicando el agarre [18], aumentando la probabilidad de que el objeto se resbale y provoque un accidente [14], por lo que según una investigación realizada [19], las posturas forzadas son uno de los factores de riesgo asociadas al levantamiento y transporte manual de cargas, principales actividades desarrollas por los trabajadores del Mercado.

Condiciones del entorno: Con respecto al entorno, según las visitas realizadas a los locales de trabajo se observó que el piso, en algunas ocasiones es resbaladizo (húmedo) o desigual, el acceso a las áreas de almacenamiento a los locales es reducido, los pasillos no cumplen con las dimensiones mínimas establecidas en Reglamento a la Ley de igualdad de oportunidades para personas con discapacidad [20], que establece una dimensión de 1,2 metros para los pasillos de uso común; además el espacio de trabajo para algunos de los locales no cumple 
con $2 \mathrm{~m}^{2}$ libres por trabajador, siendo inferior a lo establecido en nuestra legislación [21], ni la altura que es inferior a dos metros y medio; por lo que la condición actual provoca que se tomen posturas incómodas.

Evaluación de las operaciones que involucran un manejo manual de cargas que influyen en la ocurrencia de lesiones músculo esqueléticas:

Tablas MAC: Con este instrumento se evaluaron 6 de los 17 puestos (eligiendo uno por tipo de local, debido a que realizan las mismas actividades), en donde los traslados de los materiales comprometen diversas partes del cuerpo de los trabajadores como lo son la espalda, piernas, brazos y manos, además, de que no se utilizan ayudas mecánicas para realizar estas tareas.

En el cuadro 1 se indica el resumen de los datos obtenidos con este instrumento.

Cuadro 1. Resultados finales según método MAC

\begin{tabular}{|c|c|c|c|c|}
\hline \multirow{2}{*}{ Número de Local / oficio } & \multicolumn{3}{|c|}{ Puntuación numérica total por tarea } & \multirow{2}{*}{ Total } \\
\cline { 2 - 4 } & Levantar & Equipo & Transportar & \\
\hline Local 128, Carnicería & 22 & & 22 & 66 \\
\hline Local 82 Abastecedor & 17 & 17 & 17 & 51 \\
\hline Local 170 Pescadería & 14 & 14 & 14 & 42 \\
\hline Local 66 Verdulería & 15 & 12 & 15 & 42 \\
\hline Local 24 Huevos y Pollos & 10 & 10 & 12 & 32 \\
\hline Local 10 Productos Agrícolas & 13 & 10 & 9 & 32 \\
\hline
\end{tabular}

Los casos en donde el valor resultó más alto corresponden a las labores realizadas en la carnicería y abastecedor. Específicamente para la carnicería, de las actividades evaluadas, el acarreo de carne resultó con mayor puntaje debido a los siguientes factores: la postura de la espalda del trabajador al momento de traslado de las piezas de carne, la distancia de recorrido que el trabajador realiza (en promedio 180 metros, la cual varía dependiendo de donde se estacione el camión que les provee la mercadería en la periferia del Mercado Municipal), desde el exterior del mercado hacia el local. Bajo esta condición, se presenta un aumento de la fuerza de tensión en el disco vertebral [22], viéndose comprometido el sistema cardiovascular, debido a que a distancias de más de $50 \mathrm{~m}$ el consumo de oxígeno y la frecuencia cardiaca aumentan [11], sumado a esto, se cuenta con pesos manipulados en algunos casos superiores a 150 kilos colocados en la espalda, que según una investigación realizada en trabajadores de mercados mayoristas en Perú, los sacos con estos pesos ocasionan que el proceso de trabajo sea riesgoso para la salud de las personas expuestas, debido al peso extremadamente excesivo [23]. Lo que podría aumentar la probabilidad de un accidente laboral en el traslado, así como incrementar las lesiones, debido a que [24], el manejo y levantamiento de cargas son las principales causas de lumbalgia y hernias de disco [25], que conllevan a periodos prolongados de recuperación. En muchas ocasiones, porque cuando la persona es consciente del problema, la lesión por lo general está en un estado avanzado de evolución [26]. Incluso, como se indicó anteriormente, la zona más afectada manifestada por los trabajadores corresponde a la espalda dorsal, seguida del cuello, hombros, piernas y espalda lumbar, lo cual se puede comparar con los datos reportados en una investigación realizada [23], en donde la lumbalgia y la hiperxifosis dorsales son las patologías más frecuentes, relacionadas con el manejo de cargas. 
En el local denominado abastecedor, los trabajadores realizan la tarea de traslado de sacos que contienen granos, llevándoles en la espalda, por razones como la rapidez para la realización de la tarea, además el poco espacio en pasillos y al almacenamiento de productos en las zonas de paso, lo que les dificulta el traslado de los mismos, comprometiendo el desplazamiento y la agilidad del trabajador. En el siguiente apartado sobre la fuerza relacionada con la tarea, se podrá apreciar como la misma resulta afectada debido a las actividades que se desarrollan.

Fuerza relacionada con la tarea: Se indican en el cuadro 2 los resultados de todas las evaluaciones realizadas con el dinamómetro a los trabajadores participantes en el estudio. En todos los casos evaluados la mano predominante es la derecha, sin embargo, las comparaciones de los resultados con lo establecido en los datos normativos para agarre y fuerza de pinza, se realizaron para ambas manos.

En el $82 \%$ de los casos, el valor al inicio de la jornada es mayor en ambas manos que al finalizar la misma, lo que demuestra que la fatiga al realizar la tarea y la condición en la que se desarrolla, son factores importantes que afectan la fuerza [27]. En el caso de la comparación de la fuerza con el valor recomendado en la mano según la edad, solamente se supera en un 29\% de los casos, situación que podría ocasionar una mayor fatiga en el trabajador, debido a que se estaría realizando un esfuerzo mayor al levantar las cargas.

Los cuatro trabajadores de la Carnicería, presentan datos de fuerza de presión mayor al valor recomendado, lo que podría deberse a que ellos constantemente ejercitan sus manos utilizándolas para el corte y destace de la carne, además de que son los que manejan pesos mayores como se comentó anteriormente, un dato interesante es que el mayor esfuerzo lo realizan solamente en las dos primeras horas de la jornada de trabajo, momento en que trasladan y destazan la carne en los diversos cortes para la venta. De igual manera sucede con el trabajador 6 de pescadería, que cuenta con 18 años, el cual supera el parámetro de referencia, por lo que se podría pensar que el factor edad podría ser favorable por ser más joven que el resto, ya que la capacidad de trabajo físico disminuye conforme aumenta la edad [28].

Consumo metabólico relacionado con la tarea: Se obtuvo mediante la conversión de la frecuencia cardiaca a carga metabólica [13], alcanzando valores en el caso de un trabajador de 6004 kcal. durante su jornada laboral.

De acuerdo a "Cortés Díaz [29]",el metabolismo para actividades laborales que suponen la realización de esfuerzo estáticos y dinámicos (como en el caso de los trabajadores del Mercado) está comprendido entre 2000 y $2500 \mathrm{kcal} /$ día, por lo que en el 35\% de los casos evaluados se alcanza ese valor o se sobrepasa, lo que podría generar fatiga.

Con base en esta información, se procedió a establecer la tasa metabólica para cada uno de los trabajadores, para luego comparar con el Anexo A de la UNE EN ISO 8996, que establece ese dato para diferentes ocupaciones. Los resultados mayores fueron obtenidos para los puestos de carnicería con valores que van de los 268 a 334.4 W/M².

Para clasificar la ocupación según lo establecido en la norma indicada anteriormente, Tabla A.1, se utiliza la referencia de dependiente de comercio, que establece un valor de 100 a 120 $\mathrm{W} / \mathrm{m}^{2}$, y el de carnicero 105 a $140 \mathrm{~W} / \mathrm{m}^{2}$, por lo que según los resultados obtenidos solamente cuatro de los casos analizados (24\%) se mantienen por debajo de esa tasa metabólica, para el resto de los puestos se supera, situación que se puede deber a que las actividades que los trabajadores realizan involucran no solamente el vender el producto, sino también como se ha comentado, el manipular las cargas por lugares externos a su puesto de trabajo.

Al comparar los valores obtenidos en la tasa metabólica con la Tabla A.2 de la norma de referencia se obtiene que, según el tipo de actividad los trabadores de carnicería tienen una tasa metabólica alta, asociada con trabajo intenso con brazos y tronco [13]. 
Cuadro 2. Datos de los trabajadores evaluados con dinamómetro antes y después de manipular cargas

\begin{tabular}{|c|c|c|c|c|c|c|c|}
\hline $\begin{array}{l}\text { Número de } \\
\text { trabajador }\end{array}$ & Edad & Género & Tipo de local & $\begin{array}{l}\text { Momento } \\
\text { de la } \\
\text { jornada }\end{array}$ & $\begin{array}{l}\text { Mano der. } \\
\text { (Fuerza) }\end{array}$ & $\begin{array}{c}\text { Mano izq } \\
\text { (Fuerza) }\end{array}$ & $\begin{array}{c}\text { Valor recomendado } \\
\text { mano (dominante/ } \\
\text { No dominante) } \\
\text { (libras) }\end{array}$ \\
\hline \multirow{2}{*}{1} & \multirow{2}{*}{49} & \multirow{2}{*}{ M } & \multirow{2}{*}{ Verdulería } & Inicio & 110 libras & 88 libras & \multirow{2}{*}{$109,9 / 100,8$} \\
\hline & & & & Final & 93 libras & 88 libras & \\
\hline \multirow{2}{*}{2} & \multirow{2}{*}{27} & \multirow{2}{*}{$M$} & \multirow{2}{*}{ Pescadería } & Inicio & 110 libras & 88 libras & \multirow{2}{*}{$120,8 / 110,5$} \\
\hline & & & & Final & 99 libras & 79 libras & \\
\hline \multirow{2}{*}{3} & \multirow{2}{*}{26} & \multirow{2}{*}{ M } & \multirow{2}{*}{ Pollo-huevos } & Inicio & 73 libras & 66 libras & \multirow{2}{*}{$120,8 / 110,5$} \\
\hline & & & & Final & 88 libras & 77 libras & \\
\hline \multirow{2}{*}{4} & \multirow{2}{*}{41} & \multirow{2}{*}{ M } & \multirow{2}{*}{ Soda } & Inicio & 77 libras & 84 libras & \multirow{2}{*}{$116,8 / 112,8$} \\
\hline & & & & Final & 86 libras & 90 libras & \\
\hline \multirow{2}{*}{5} & \multirow{2}{*}{54} & \multirow{2}{*}{$M$} & \multirow{2}{*}{ Verdulería } & Inicio & 66 libras & 55 libras & \multirow{2}{*}{$113,6 / 101,9$} \\
\hline & & & & Final & 66 libras & 59 libras & \\
\hline \multirow{2}{*}{6} & \multirow{2}{*}{17} & $M_{1}$ & Pecondarín & Inicio & 140 libras & 120 libras & $121 / 1015$ \\
\hline & & $\mid \mathrm{VI}$ & rescadenta & Final & 130 libras & 118 libras & $121 / 104,5$ \\
\hline 7 & 38 & $M$ & Productos & Inicio & 115 libras & 98 libras & $1197 / 1129$ \\
\hline 1 & 38 & $\mid \mathrm{VI}$ & agrícolas & Final & 100 libras & 80 libras & $119, \pi / 112,9$ \\
\hline $8^{*}$ & 12 & $M$ & Ahpeteredor & Inicio & 25 libras & 70 libras & $1168 / 1128$ \\
\hline 0 & $4<$ & IVI & ANdSIELEUI & Final & 38 libras & 77 libras & $110,0 / 11<, 0$ \\
\hline$a$ & 30 & $\mathrm{~F}$ & Seda & Inicio & 60 libras & 55 libras & 787168 \\
\hline$y$ & 30 & $\Gamma$ & sodid & Final & 57 libras & 50 libras & $10,1 / 00$ \\
\hline 10 & 12 & $\mathrm{~F}$ & Sond & Inicio & 52 libras & 45 libras & 701602 \\
\hline 10 & 42 & & sodid & Final & 48 libras & 40 libras & $10,4 / 02,3$ \\
\hline 11 & 20 & $M$ & Productos & Inicio & 85 libras & 87 libras & $1107 / 1120$ \\
\hline 11 & 30 & IVI & agrícolas & Final & 85 libras & 78 libras & $119, / / 112,9$ \\
\hline 10 & 10 & $M$ & Corningrín & Inicio & 183 libras & 176 libras & $1160 / 1120$ \\
\hline 12 & 40 & $\mid \mathrm{VI}$ & carmicenta & Final & 176 libras & 172 libras & $110,8 / 112,8$ \\
\hline 10 & 50 & $M$ & Corninorís & Inicio & 150 libras & 106 libras & $1011 / 0020$ \\
\hline 13 & 58 & $\mid \mathrm{M}$ & carniceria & Final & 141 libras & 99 libras & $101,1 / 83,2$ \\
\hline 11 & 15 & $M$ & Cornirorín & Inicio & 181 libras & 137 libras & $1009 / 1008$ \\
\hline 14 & 45 & $\mid \mathrm{M}$ & carniceria & Final & 172 libras & 128 libras & $109,9 / 100,8$ \\
\hline 15 & 65 & $M$ & Cornicorín & Inicio & 133 libras & 133 libras & $011 / 768$ \\
\hline 15 & 65 & $\mid \mathrm{M}$ & Carniceria & Final & 128 libras & 121libras & $91,1 / / 6,8$ \\
\hline & & - & & Inicio & 33 libras & 25 libras & \\
\hline 16 & 5 & $r$ & soda & Final & 30 libras & 20 libras & $5 /, 3 / 4 /, 3$ \\
\hline 17 & 50 & $M$ & Yordularín & Inicio & 72 libras & 65 libras & $1120 / 1010$ \\
\hline 18 & 52 & $\mathrm{IVI}$ & verauleria & Final & 70 libras & 58 libras & (1/3,6/IU1,9 \\
\hline & & & Nota:" & ión en $\mathrm{m}$ & derecha & & \\
\hline & & & olor azul: trab & dores que & peran los pa & netros & \\
\hline
\end{tabular}




\section{Evaluación de riesgos}

Se procedió a elaborar una matriz de riesgo, en donde se integra toda la información, con el objetivo de priorizar los riesgos a los que se exponen los trabajadores del mercado.

Como se puede observar en el cuadro 3, en las tareas de levantamiento y traslado de cargas, así como en las de acomodo de producto, es donde se presentan los riesgos no aceptables (nivel de riesgo I), que generan peligros como posturas inadecuadas o prolongadas durante la jornada, los cuales pueden tener efectos como lumbalgias y problemas circulatorios. Además, el traslado de las cargas, al realizarse en espacios tan reducidos y con productos al hombro en muchas ocasiones, se pueden generar cervicalgias, o bien consecuencias relacionadas con el entorno por donde se transita como caídas y golpes; por lo cual según la norma INTE de referencia [30], son los que requieren de una intervención inmediata para disminuir el nivel de riesgo, correspondiendo a una situación crítica.

Cuadro 3. Matriz de riesgos

\begin{tabular}{|c|c|c|c|c|c|c|c|c|c|c|c|}
\hline \multirow[b]{2}{*}{ Tareas } & \multicolumn{2}{|c|}{ Peligro } & \multirow[b]{2}{*}{$\begin{array}{l}\text { Efectos } \\
\text { posibles }\end{array}$} & \multicolumn{7}{|c|}{ Evaluación del riesgo } & \multirow{2}{*}{$\begin{array}{c}\text { Valoración } \\
\text { del riesgo }\end{array}$} \\
\hline & Descripción & Clasificación & & ND & NE & NP & INP & NC & $\begin{array}{c}\text { NR e } \\
\text { intervención }\end{array}$ & $\begin{array}{c}\text { Interpretación } \\
\text { de NR }\end{array}$ & \\
\hline $\begin{array}{c}\text { Levantamiento } \\
\text { de cargas }\end{array}$ & $\begin{array}{c}\text { Posturas } \\
\text { inadecuadas }\end{array}$ & Biomecánico & Lumbalgias & 10 & 3 & MA-30 & $\begin{array}{l}\text { Muy } \\
\text { alto }\end{array}$ & 60 & 1800 & I & No \\
\hline $\begin{array}{l}\text { Traslado de } \\
\text { cargas }\end{array}$ & $\begin{array}{l}\text { Manejo de } \\
\text { cargas por } \\
\text { espacios } \\
\text { reducidos }\end{array}$ & $\begin{array}{l}\text { Biomecánico } \\
\text { y seguridad }\end{array}$ & $\begin{array}{l}\text { Lumbalgias y } \\
\text { cervicalgias. } \\
\text { Caídas y } \\
\text { golpes }\end{array}$ & 10 & 2 & A-20 & Alto & 60 & 1200 & I & No \\
\hline $\begin{array}{l}\text { Corte y } \\
\text { destace* }\end{array}$ & $\begin{array}{l}\text { Movimientos } \\
\text { repetitivos, } \\
\text { posturas } \\
\text { incómodas } \\
\text { y manejo } \\
\text { inadecuado } \\
\text { de }\end{array}$ & $\begin{array}{c}\text { Biomecánico } \\
\text { y mecánico }\end{array}$ & $\begin{array}{c}\text { Síndrome } \\
\text { del túnel } \\
\text { de carpo, } \\
\text { tendinitis. } \\
\text { Heridas }\end{array}$ & 6 & 2 & A-12 & Alto & 25 & 300 & II & No \\
\hline $\begin{array}{l}\text { Venta de } \\
\text { producto }\end{array}$ & $\begin{array}{c}\text { Posturas } \\
\text { prolongadas } \\
\text { (según local } \\
\text { estáticas o } \\
\text { dinámicas) }\end{array}$ & Biomecánico & $\begin{array}{c}\text { Lumbalgias, } \\
\text { problemas } \\
\text { circulatorios }\end{array}$ & 2 & 4 & M-8 & Medio & 10 & 80 & III & Sí \\
\hline
\end{tabular}

Nota: ND: Nivel de deficiencia. NE: Nivel de exposición. NP: Nivel de probabilidad. INP: Interpretación del nivel de probabilidad. NC: Nivel de Consecuencia. NR: Nivel de riesgo.

Los riesgos medios (nivel de riesgo II) se encontraron en las actividades corte y destace (que corresponden a tareas que se realizan únicamente en los locales de pescadería y carnicería), pues en estos se generan movimientos repetitivos, posturas incómodas, un manejo inadecuado 
de las herramientas que utilizan, así como el piso húmedo; además hay contacto con la sangre de los animales; todas estas condiciones pueden producir efectos a la salud tales como: síndrome del túnel carpal y tendinitis, enfermedades relacionadas por el contacto con los animales, además de caídas, heridas y golpes. Estos riesgos deben ser corregidos pues se clasifican como no aceptables al no tener controles establecidos.

\section{Conclusiones}

En los locales evaluados en el Mercado Municipal de Cartago, todos los trabajadores realizan manejo manual de cargas, que se relaciona a las molestias manifestadas a nivel de espalda dorsal, lumbar, cuello, hombros, y piernas, que podrían generar lesiones músculo esqueléticas si no se llegan a establecer controles adecuados.

En las actividades que se realizan, existen elementos como el peso, la forma y tamaño de las cargas, las distancias de recorrido, así como las características de los locales y de los trabajadores, que se convierten en factores de riesgo asociados a las operaciones de transporte, levantamiento y acomodo de los productos, que según la evaluación realizada se clasifican como críticos (Nivel I).

El principal riesgo corresponde al biomecánico, debido a peligros como las posturas inadecuadas y prolongadas, manejo de cargas por espacios reducidos y en locales con dimensiones insuficientes, aunados a las características personales como la escasa actividad física, y un alto consumo metabólico producto de la forma en las que se realizan las tareas, en donde predomina el esfuerzo muscular dinámico.

\section{Agradecimientos}

Este proyecto fue desarrollado gracias al financiamiento recibido por parte de la Vicerrectoría de Investigación y Extensión, del Instituto Tecnológico de Costa Rica.

Se agradece la colaboración de la Municipalidad de Cartago, especialmente al señor Guillermo Rodríguez Hernández quien brindó todo el apoyo logístico durante la ejecución del mismo y a los trabajadores de los locales participantes.

\section{Referencias}

[1] A. Luttmann, M. Jager y B. Griefahn, Prevención de transtornos musculoesquléticos en el lugar de trabajo, Berlín: Organización Mundial de la Salud, 2004.

[2] C. Ferraro, Apoyando a las PYMES: Políticas de fomento en América Latina y el Caribe., Santiago: Naciones Unidas, 2011.

[3] L. Arenas Ortiz y O. Cantú Gómez, «Factores de riesgo de transtornos músculo-esqueléticos crónicos laborales,» Medicina Interna de México, vol. 29, n 4, pp. 370-379, 2013.

[4] Y. Chen, J. Mc Donald y N. Cherry, «Incidence and suspected cause of work-related musculoskeletal disorders, United Kingdom, 1996-2001,» Occupational Medicine, vol. 56, nº 6, pp. 406-413, 2006.

[5] E. Agila Palacios, C. Colunga Rodríguez, E. González Muñoz y D. Delgado García, «Síntomas músculoesqueléticos en trabajadores operativos del área de mantenimiento de una empresa petrolera ecuatoriana, » Ciencia \& Trabajo, vol. 51, pp. 198-205, 2014.

[6] V. Córdova, R. Pinto y B. Chavéz, «Córdova, V., Pinto, R., LLambías, J., \& Chavéz, B. (2009). Capacidad de manejo manual de carga de trabajadores Chilenos: pesos máximos aceptables para tareas de levantamiento., » Ciencia \& Trabajo, vol. 34, pp. 204-210, 2009.

[7] A. Creus Solé y J. Mangosio, Seguridad e Higiene en el Trabajo, Buenos Aires: Alfaomega, 2011.

[8] P. Buckle y J. Devereux, «The nature of work-related neck and upper limb musculoskeletal disorders.,» Applied ergonimics, vol. 33, n 3, pp. 207-217, 2002. 
[9] R. Rosario Amézquita y T. Améz, «Prevalencia de trastornos músculo-esqueléticos en el personal de esterilización,» Medicina y seguridad del trabajo, pp. 24-43, 2014.

[10] Ministerio de Trabajo y Seguridad Social, «Estadísticas de Salud Ocupacional Costa Rica 2015,» Consejo de Salud Ocupacional, San José, 2015.

[11] S. Konz, Diseño de sistemas de trabajo. México: LIMUSA S.A., México: LIMUSA S.A, 1990.

[12] V. Mathiowetz, N. Kashman, G. Volland, K. Weber, M. Dowe y S. Rogers, «Grip and Pinch Strength: Normative Data for Adults,» Arch Phys Med Rehabil, pp. 69-72, 1985.

[13] AENOR, Ergonomía del ambiente térmico. Determinación de la tasa metabólica (ISO 8996: 2004), Madrid: ISO, 2005.

[14] Agencia Europea para la seguridad y salud en el trabajo, «Riesgos asociados a la manipulación manual de cargas en el lugar de trabajo., 2007.

[15] J. Pérez Bilbao y C. Nogareda Cuixart, NTP 367: Envejecimiento y trabajo: la gestión de la edad., Madrid: INSHT. 1992.

[16] J. F. Ramírez Villada, C. E. Melo y R. G. Laverde, «Análisis de la relación entre la potencia, la capacidad anaeróbica y la velocidad desplazamiento en varones entre 50 y 60 años de edad.,» 2009. [En línea]. Available: http://www.archivosdemedicina.com/medicina-de-familia/anlisis-de-la-relacin-entre-la-potencia-la-capacidadanaerbica-y-la-velocidad-desplazamiento-en-varones-entre-50-y-60-aos-de-edad.pdf. [Último acceso: 28 Junio 20017].

[17] M. López Alonso, M. Martínez Aires y E. Martín González, «Análisis de los riesgos musculoesqueléticos asociados a los trabajos de ferrallas. Buenas prácticas.» 2011.

[18] Universidad de Málaga, Manipulación manual de cargas: Factores de riesgo que están presentes y forma de prevenir los riesgos asociados., Servicio de Prevención de Riesgos Laborales (Sepruma)., 2007.

[19] A. Caicedo G, J. Manzano, D. Gómez-Vélez y L. Gómez, «Factores de Riesgo, Evaluación, Control y prevención en el Levantamiento y Transporte Manual de Cargas,» Revista Colombiana de Salud Ocupacional, pp. 5-9, 2015.

[20] Poder Ejecutivo, Reglamento a la Ley de Igualdad de oportunidades para personas con discapacidad. san José: La Gaceta., San José: La Gaceta, 1998.

[21] Ministerio de Trabajo, Reglamento General de Seguridad e Higiene del Trabajo, San José: La Gaceta, 1970.

[22] S. Nogareda Cuixart y M. Canosa Bravo, «NTP 477: Levantamiento manual de cargas: ecuación del NIOSH,» INSHT, Madrid, 1998.

[23] L. Vigil, R. Gutiérrez, W. Cáceres, H. Collantes y J. Beas, «Salud ocupacional en el trabajo de estiba: los trabajadores de mercados mayoristas de Huancayo, 2006.," Revista Peruana de medicina experimental y salud pública,, pp. 336-342, 2007.

[24] S. Nogareda Cuixart y M. Canosa Bravo , NTP 477: Levantamiento manual de cargas: ecuación del NIOSH, Madrid: INSHT, 1998.

[25] D. González Maestre, «Ergonomía y Psicosociología,» Fundación Confemetal., Madrid. 2008.

[26] Egarsat, «Manipulación manual de cargas.,» Ministerio de empleo y seguridad social., Madrid, 2015.

[27] L. Muñoz Jashimoto, E. Vega Bustillos, F. Lopez Millan, B. Ortiz Navar y K. Duarte, «Fuerza máxima de agarre con mano dominante y no dominante. Hermosillo., " Hermosillo, 2009.

[28] P. Mondelo, E. Torada, S. Comas Úriz, E. Castejón Vilella y E. Bartolomé Lacambra, «Ergonomía 2. Confort y estrés térmico. México: Alfaomega.,» Alfaomega, México, 2008.

[29] J. Cortés Díaz, Seguridad e Higiene del trabajo, Madrid: TÉBAR, 2012.

[30] INTECO, Guía para la identificación de los peligros y la evaluación de los riesgos de salud y seguridad ocupacional., San José, 2011.

[31] Organización Mundial de la Salud, «Índice UV. Solar Mundial. Guía práctica,» OMS, 2003.

[32] C. Young, "Solar ultraviolet radiation and skin cancer,» Occupational Medicine, pp. 82-88, 2009.

[33] A. M. Siani, G. R. Casale, A. Colosimo, C. A. Lang y M. G. Kimlin, «Occupational exposure to solar ultraviolet radiation of vineyard workers in Tuscany (Italy),» Photochemistry and Photobiology, pp. 925-934, 2011.

[34] E. F. Bustamante, «El uso adecuado de protectores solares en Costa Rica,» Revista Costarricense de Ciencias Médicas, 1999. 
[35] K. Heinrich Bettoni, R. Pacheco Cubero, M. Solera Flores y M. Alfaro Hernandez, «Series de brillo solar en Costa Rica,» Ministerio de Ambiente, Energía, San José, 2013.

[36] L. Vigil, R. Gutiérrez, W. Cáceres, H. Collantes y J. Beas, «Salud ocupacional en el trabajo de estiba: los trabajadores de mercados mayoristas de Huancayo 2006, "Revista Peruana de medicina experimental y salud pública,, pp. 336-342, 2007. 